\title{
Developing a Taxonomy of Dark Triad Triggers at Work - A Grounded Theory Study Protocol
}

\author{
Annika Nübold ${ }^{1 *}$, Josef Bader ${ }^{2}$, Nera Bozin ${ }^{3}$, Romil Depala ${ }^{4}$, Helena Eidast ${ }^{5}$, \\ Elisabeth A. Johannessen ${ }^{6}$ and Gerhard Prinz ${ }^{7 *}$
}

${ }^{1}$ Department of Work and Social Psychology, Maastricht University, Maastricht, Netherlands, ${ }^{2}$ Faculty of Psychology and Educational Sciences, University of Coimbra, Coimbra, Portugal, ${ }^{3}$ Department of Psychology, University of Ljubljana, Ljubljana, Slovenia, ${ }^{4}$ Department of Experimental Psychology, University of Oxford, Oxford, UK, ${ }^{5}$ Independent Researcher, Tallinn, Estonia, ${ }^{6}$ Department of Psychology, University of Winchester, Winchester, UK, ${ }^{7}$ Department of Basic Psychological Research and Research Methods, University of Vienna, Vienna, Austria

\section{OPEN ACCESS}

Edited by:

Rocio Del Pino,

University of Deusto, Spain

Reviewed by:

Serge Brand,

University of Basel, Switzerland

Peter Karl Jonason,

Western Sydney University, Australia

*Correspondence:

Annika Nübold

a.nubold@maastrichtuniversity.nl

Gerhard Prinz

gerhard.prinz@univie.ac.at

Specialty section:

This article was submitted to

Quantitative Psychology

and Measurement,

a section of the journal

Frontiers in Psychology

Received: 11 June 2016

Accepted: 15 February 2017

Published: 07 March 2017

Citation:

Nübold A, Bader J, Bozin N,

Depala $R$, Eidast $H$, Johannessen EA and Prinz G (2017) Developing

a Taxonomy of Dark Triad Triggers

at Work - A Grounded Theory Study

Protocol. Front. Psychol. 8:293.

doi: 10.3389/fpsyg.2017.00293
In past years, research and corporate scandals have evidenced the destructive effects of the dark triad at work, consisting of narcissism (extreme self-centeredness), psychopathy (lack of empathy and remorse) and Machiavellianism (a sense of duplicity and manipulativeness). The dark triad dimensions have typically been conceptualized as stable personality traits, ignoring the accumulating evidence that momentary personality expressions - personality states - may change due to the characteristics of the situation. The present research protocol describes a qualitative study that aims to identify triggers of dark triad states at work by following a grounded theory approach using semi-structured interviews. By building a comprehensive categorization of dark triad triggers at work scholars may study these triggers in a parsimonious and structured way and organizations may derive more effective interventions to buffer or prevent the detrimental effects of dark personality at work.

Keywords: dark triad, personality states, workplace triggers, taxonomy, grounded theory

\section{INTRODUCTION}

The study of dark personality and its impact in the workplace has gained increasing attention in the past years (Spain et al., 2014). Dark personality traits are defined as characteristics that reflect a motivation to elevate the self and harm others (Paulhus and Williams, 2002). Amongst other conceptualizations (e.g., Hogan and Hogan, 2001) the dark triad consisting of narcissism, psychopathy, and Machiavellianism (Paulhus and Williams, 2002) represents the most popular operationalization of dark personality at work (Spain et al., 2014).

Narcissism is characterized by feelings of grandiosity, entitlement, dominance, and superiority (Spain et al., 2014). People who show this trait tend to be charming or pleasant in the short term while in the long run presenting difficulty in maintaining successful interpersonal relationships, lacking trust and care for others (Morf and Rhodewalt, 2001). Psychopathy involves feelings of impulsivity, thrill-seeking, low empathy and anxiety. Those that present a psychopathic trait seek immediate gratification of their needs, lack guilt and conscience, being less prone to experience embarrassment and failing to learn from punishment for misdeeds (Hare, 1985). Machiavellianism is associated with cynicism, low affect, an unconventional view of morality and a focus exclusively on personal goals (Christie and Geis, 1970). Thus, those who exemplify this trait tend to be exceedingly willing to manipulate others and take a certain pleasure in successfully deceiving them (Jones and Paulhus, 2014). 
Importantly, despite similarities and overlap, the dark triad is not identical with clinically relevant personality disorders nor does it reflect simply extreme forms of normal personality traits (Harms et al., 2014). Both research and past corporate scandals have evidenced the dark triad's effects on counterproductive work behaviors (e.g., O’Boyle et al., 2012; Spain et al., 2014) and a variety of other destructive outcomes, such as heightened competitiveness, dysfunctional job crafting, and corruption (Carter et al., 2014; Roczniewska and Bakker, 2016; Zhao et al., 2016).

To date, research has mainly focused on the detrimental outcomes of dark personality. Although initial efforts have been made in past years to discover the psychological underpinnings of the dark triad (Paulhus and Williams, 2002), research has failed to address the role of situational factors in eliciting momentary expressions of dark personality characteristics. To date, the dark triad has exclusively been conceptualized and investigated in its trait-like form, ignoring evidence for the malleability and short-term fluctuation of personality states, the expression of one's personality in a specific moment (e.g., Fleeson, 2001). Due to the predominant view of the dark triad as stable traits, situational cues eliciting dark triad behavior have not been of concern so far. Attempts to identify the roots of dark triad personality have thus focused on very broad, generic explanations, such as evolutionary (e.g., Jonason et al., 2009), behavioral genetic (e.g., Vernon et al., 2008), socio-ecological (Jonason et al., 2016), neuro-biological (Jonason and Jackson, 2016), and motivational (Harms et al., 2014; Jonason and Ferrell, 2016) foundations.

In our study we apply a more dynamic approach to dark personality. Drawing on interactionist models of personality, such as the cognitive affective personality system (CAPS; Mischel and Shoda, 1995, 1998) or whole trait theory (Fleeson and Jayawickreme, 2015) that build upon Lewin's (1936) equation for predicting behavioral reactions $[B=f(P \times E)]$, we assume that stable personality traits $(P)$ interact with environmental characteristics $(E)$ to produce a specific behavioral response $(B)$, or in other words, a specific personality state (see Figure 1 for our conceptual model; please note that the listed situations at work merely represent examples of potential triggers and don't reflect empirical findings). Mischel and Shoda $(1995,1998)$ called this complex interplay if-then contingencies describing the idea that specific situational cues make people reliably react in a specific way, based on their personality traits. Empirical studies (e.g., Judge et al., 2013) and entire special issues (Beckmann and Wood, 2016) have evidenced that the behavioral expressions (i.e., personality states) in relation to changing situations present a potentially predictable reflection of personality (Mischel and Shoda, 1995, 1998).

Despite the growing evidence that personality expressions are dependent on situational cues, research on the triggering function of job characteristics is still in its infancy and has only focused on adaptive personality states so far (Judge et al., 2013; Dóci and Hofmans, 2015). The usual approach for adaptive personality states to conceptualize situations mainly as opportunities to express one's personality (Ten Berge and De Raad, 1999) may, however, be problematic for the concept of the dark triad. Whereas some situations may allow for or even encourage the expression of dark personality characteristics because they may be functional in that moment (e.g., being self-aggrandizing in a selection interview), other situations may rather trigger dark personality states because specific needs and motives are not fulfilled (e.g., the need for power). Thus, although "a psychology of situations has begun to take shape" (Funder, 2016, p. 203), a comprehensive taxonomy of situational triggers of specific personality characteristics, including the dark triad, has yet to be established.

In this study, we aim to identify the underlying situational antecedents in the work environment (E) that lead to
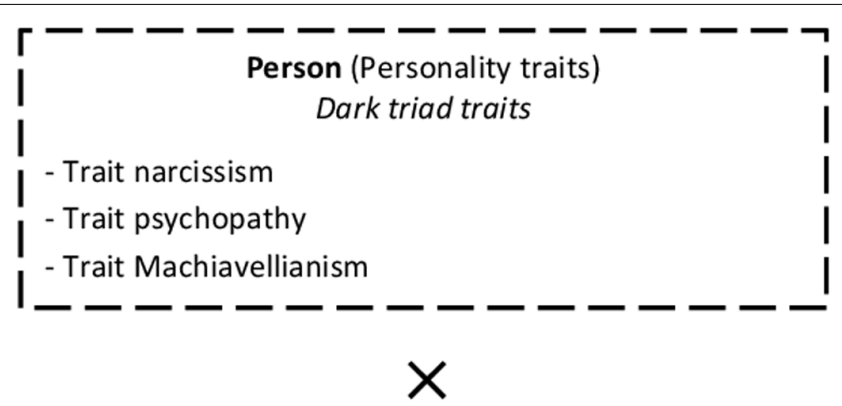

Environment (Situations) Situations at work (potential triggers)

- Role modelling (competitive or antisocial context)

- Coping with stress (time pressure, role conflict)

- Reacting to threat (abusive supervision)
Behavior (Personality states) Dark triad states

- State narcissism

- State psychopathy

- State Machiavellianism

FIGURE 1 | Conceptual model following Lewin's equation for behavior (Lewin, 1936): $B=f(P, E)$. 
within-person variation in momentary dark triad expressions, i.e., in dark triad personality states (B), that is, state narcissism, state psychopathy and state Machiavellianism, resulting in a comprehensive taxonomy of triggers. We use a grounded theory approach (Strauss and Corbin, 1998; Corbin and Strauss, 2008) as this qualitative methodology is particularly suited for complex social processes about which little is known yet (Glaser and Strauss, 1967; Willig, 2009). Identifying potential triggers of dark personality expressions at work and building taxonomy of these triggers is important in several ways. On a theoretical level, a more comprehensive understanding of the triggers of dark personality states and their potential interconnections is crucial in order to acquire knowledge on the nomological network and common mechanisms that may be associated with specific groups or categories of triggers. Moreover, identifying triggers of dark personality expressions at work is practically important, as organizations strongly benefit from detailed knowledge on situations that may "make people snap." Organizations and Human Resource (HR) professionals will be able to design evidence-based interventions that help prevent employees from expressing their dark tendencies which may bring harm to organizations and their members over the short and long term. These actions may not only include job design and selective placement, but also training and coaching interventions that sensitize employees for potentially dangerous situations and helping them to either themselves better manage and regulate their dark impulses or increase their ability to cope with others' dark behaviors, enabling better relationships and work ethics within their organizations.

With the present study, we contribute to the literature in three important ways: Firstly, identifying triggers of dark personality expressions at work adds to the evolving personality state literature by broadening the domain of situational predictors and allowing us to better understand the complex interplay between person and situation characteristics $(P \times E)$ jointly leading to the expression of personality states (B) in a specific situation. Secondly, our taxonomy may be used to further advance research in the field of dark personality at work by stimulating the creation of instruments such as questionnaires or situational judgment tests that enable scholars to study these triggers in quantitative studies in a more standardized and parsimonious way. In addition, our taxonomy may further add to the study of long-term personality development, helping to further explore how individuals' personality turns dark over time by investigating how short-term dynamics add up in a longitudinal fashion (Hogan et al., 1994). Finally, editors of top tier journals (e.g., Suddaby, 2006; Bansal and Corley, 2011) explicitly acknowledge the value of grounded theory and call for more qualitative research in the organizational literature. By following this call we promote a methodology that is particularly useful for examining situated processes like employee interactions in complex organizational settings (Locke, 2001). Qualitative research complements the many quantitative studies in the organizational literature by offering the reader a close-up of the phenomenon being studied and providing the opportunity to raise new research questions, revealing "deeper insights into management, organizations, and society, which are critical to understanding and potentially shaping our world" (Bansal and Corley, 2011, p. 235).

In sum, our research questions are as follows:

(1) Which situational characteristics at work trigger expressions of state narcissism?

(2) Which situational characteristics at work trigger expressions of state psychopathy?

(3) Which situational characteristics at work trigger expressions of state Machiavellianism?

\section{MATERIALS AND EQUIPMENT}

\section{Semi-structured In-depth Interviews}

In order to gather information on momentary experiences of dark triad states and their eliciting factors at work, we will conduct semi-structured in-depth interviews with employees. Semi-structured interviews enable the interviewer to flexibly adapt and add further questions depending on the answers given by the interviewee allowing for more in-depth explanations of how the person experienced the situation, thereby increasing the validity of the interview (McLeod, 2014).

Interviews will be conducted with jobholders of all seniority levels reporting about their own experiences (i.e., self-reports) or about the behavior of someone else (i.e., observer-reports), to account for different perspectives and to create a multifaceted view on the phenomenon of interest (Glaser and Strauss, 1967; Bluhm et al., 2011). As we expect it to be more difficult to obtain answers from participants about their own dark behaviors due to the possibility of socially desirable responding, we will first invite participants to talk about their own behaviors before also offering them the option of reporting the behaviors of a significant other (e.g., a colleague, supervisor or subordinate). Although self-reports are particularly valuable as they can target internal emotions and cognitions, it is important to note that research has evidenced that dark triad characteristics (e.g., features of psychopathy) can also be reliably and validly detected by lay raters, particularly if they involve interpersonal behaviors (Fowler et al., 2009). Therefore, employees reporting on their impressions and observations of a relevant situation involving another person are valuable sources of information as well (Fowler et al., 2009).

Although it has also been recommended to apply several methods of data collection (Bluhm et al., 2011), conducting observations and analyzing existing written materials will not be the focus of our research. As our study's purpose is to identify the (to date unknown) triggering factors of dark triad states, it will neither be possible to reasonably plan specific observation periods nor to conduct observations spanning many hours or even days hoping for a potential triggering situation to occur.

The actual interview will consist of three main parts:

(1) An open and generic question about the interviewees' experiences at work and their job in general in order to allow interviewees to get comfortable with reporting about their experiences while we introduce them to the topic. 
(2) The second part will include questions about experiences of specific dark triad expressions at work. Descriptions of these dark personality expressions will be based on items from validated instruments, such as the Mach IV (Christie and Geis, 1970), the Self-Report Psychopathy Scale III (SRP-III; Hare, 1985), and the Narcissistic Personality Inventory (NPI; Raskin and Hall, 1979). These descriptions will ensure a common understanding of the focal behaviors and will stimulate interviewees to reflect on situations in which they have shown these behaviors themselves (or observed them in another person). A sample question for state Machiavellianism would be: "When thinking about your work experiences in the past 12 months - can you think about a specific situation in which you have done certain things to get someone to do what you want?". In cases where participants experience difficulties in answering the question, a prompt could be: "For instance, have you said something particularly to someone to get what you want?". Interviewees will then be asked to describe a specific situation in which they expressed this behavior in more detail, for example in terms of where the situation took place, the specific individuals involved in the interaction, and the specific actions.

(3) The third step will focus on the aim of the interviews, the potential triggering situations of dark triad expressions at work. Accordingly, interviewees will be asked their opinion on what they believe may have evoked their specific behavior, that is, the preceding situation and the specific trigger of that behavior. Interviewees will also be asked about the broader context of the situation (e.g., if there have been prior incidents with someone), as the meaning of situational triggers may change from one context to another. Participants will be asked to describe this triggering situation in as much detail as possible. As suggested by previous research (Judge et al., 2013; Jonason and Ferrell, 2016; Jonason et al., 2016), we will ask participants reporting their own experiences for both, more objective environmental events (i.e., something someone did or said) and internal events (e.g., subjective feelings, motives, or cognitions), as both events may serve as antecedents to dark triad personality states. Participants reporting the behaviors of someone else will only be asked about the more objective features of the situation and will not be asked to speculate about the potential thoughts and feelings of the target person. As noted by Funder (2016) with reference to Reis (2008), situations are always filtered through the perception of the individual who experiences them, but should nonetheless be conceptualized separately from individual construal. Likewise, Rauthmann et al. (2015) emphasize in the so called processing principle that people's experiences are based upon objective environmental events which are explicitly and/or implicitly processed by individuals, thereby providing situations with meaning. The perspective that both internal and external events may be meaningful as triggers of dark personality states is in line with a post-positivist perspective and the aim to come closer to a singular truth while acknowledging that evidence is not confined to what can be physically observed and that subjective influences exist.

\section{METHODS}

\section{Design}

In the present study we use a grounded theory approach to answer our research questions. Grounded theory captures the complexity of social processes like no other methodology, it reveals content that is highly embedded in practice, and gives researchers the possibility to describe a phenomenon in great detail (Martin and Turner, 1986; Strauss and Corbin, 1998). Most importantly, it supports theorizing in "new" areas of research (Strauss and Corbin, 1998; Birks and Mills, 2015) and allows researchers to revise the direction and framework of research in real time as soon as new information and findings emerge. Our research questions are particularly appropriate for a grounded theory approach as there is a lack of research on dark personality states and therefore also a lack of essential information on their triggers.

There are multiple philosophies regarding grounded theory methodology (e.g., Glaser, 1978; Strauss and Corbin, 1998; Corbin and Strauss, 2008; Charmaz, 2014). In the present study, we follow the epistemological approach of postpositivism which assumes that there is one truth that can be discovered, but acknowledges that individuals' perceptions are influenced by the context and that information gathered in the research process is not a neutral reflection of the truth. Thus, we approach grounded theory with the understanding that reality exists and that objectivity can be reached by discovering an emergent theory that represents this reality as accurately as possible. In line with the post-positivist approach, we follow Strauss and Corbin's (1998) assumption that a theory is discovered in the data instead of being fully constructed.

Although cross-cultural research has many benefits, conducting interviews in two different languages (English and German) may be an area of concern in qualitative studies (Squires, 2009; Nurjannah et al., 2014). As recommended for multilingual research projects (Van Nes et al., 2010), we aim to make use of the original language for as long as possible. Specifically, interviews that will be conducted in English will be transcribed and coded in English while the interviews conducted in German will be transcribed and coded in German. Only after the coding procedure, we will translate the codes and the respective text passages and sample quotes derived from the German interviews into English. In order to ensure equivalence of meaning of these translations, we will follow the translation back-translation procedure by Brislin (1970), while making use of a translator moderator (Van Nes et al., 2010). The translator moderator will be the first author who will conduct the interviews in German and at the same time is highly proficient in English. Translating the codes instead of the original transcripts ensures the authenticity of the data and quality of analysis by minimizing potential misinterpretation and loss of participants' intended meanings (Larkin et al., 2007) while at the same time being more economic and feasible (Chen and Boore, 2009). 


\section{Participants}

In order to identify triggers of dark triad expressions at work, we will approach jobholders that are either willing to report on their own behavior at work or on behavioral observations of someone else, for example a colleague, their supervisor or a subordinate. In addition, we will approach subject matter experts, such as HR consultants, who may be able to report on dark triad expressions of clients and potential triggering situations based on their work with organizations. As the dark triad (Paulhus and Williams, 2002) refers to subclinical or everyday versions of maladaptive personality, in contrast to clinically relevant disorders, dark triad characteristics may be well represented in normal populations. Thus, our target sample will consist of regular employees and professionals. Potential participants will initially be approached via the interviewers' networks (e.g., via professional business and employment-oriented social networking service).

In order to achieve high heterogeneity of data sources (i.e., a maximum amount of variance in the target behaviors and situational triggers), we plan to approach male and female employees of different ages from a wide variety of branches, jobs, positions, and hierarchy levels. Through this approach, we also aim to identify more severe situations (that may be needed to trigger individuals with low levels in the dark triad) as well as less critical situations (that may function as triggers for those with high dark triad levels). We will not determine the number of interviews (our sample size) a priori (Eisenhardt and Graebner, 2007), but will continue to collect data until a theoretical saturation point has been reached and no new relevant categories of triggers emerge (Glaser and Strauss, 1967). As qualitative research handles non-numerical information and because the right sample size depends on a number of factors, such as the variety and content of answers and the scope of the study, power calculations that are appropriate in quantitative research are not applicable here (Morse, 2000; Leung, 2015). Nonetheless, scholars have for example suggested 20-30 interviews for grounded theory (Creswell, 1998), a sample size that has also been confirmed in grounded theory studies conducted in an organizational context (Seivwright and Unsworth, 2016; Wilhelmy et al., 2016). Additionally, we will document the specific steps of the theoretical sampling process to make the choice of our eventual final sample size as transparent as possible (Nelson, 2016).

\section{Procedure}

A model of the procedural steps in our study is depicted in Figure 2.

Interviews will be conducted in English and German. All interviews will be audio recorded after obtaining permission from participants to tape-record the session. Interviews will take place face-to-face at the facilities of the university the respective interviewer is affiliated with or via telephone or videoconference calls (e.g., Skype). Telephone and videophone calls have been found to be a solid substitute for face-to-face interviews, especially in semi-structured interviews, while at the same time allowing for more efficient data collection (Sturges and Hanrahan, 2004; Berg, 2007; Sullivan, 2012).
At the beginning of each in-depth interview, participants will be informed about the purpose and general context of the study in order to ensure transparency and allow for proper consideration of participation (e.g., Wilhelmy et al., 2016). Further, individuals will be ensured of the anonymity and confidentiality of their answers and the right to withdraw from the study at any point. In order to limit recall bias, participants will be instructed to respond to our interview questions based on their work experiences within the past 12 months. At the end of each interview, participants will be encouraged to complete a short questionnaire of the dark triad traits, the SD3 (Jones and Paulhus, 2014), in order to capture their baseline level of these characteristics. The assessment of their baseline will allow for a more detailed understanding of the distribution and level of dark personality characteristics in our sample and will allow us to link this information with the descriptions of the triggering situations, enabling us to control for possible moderating effects $(\mathrm{P} \times \mathrm{E})$. Research has frequently documented that personality characteristics $(\mathrm{P})$, such as neuroticism, moderate the perception of and reaction to daily experiences (E) (e.g., stressful events; Bolger and Zuckerman, 1995; Suls and Martin, 2005) and systematically influence the level and variability of personality states (B) (Fleeson and Jayawickreme, 2015). Likewise, individuals with different levels of dark triad traits may perceive and react to situations differently (either in terms of the quality and character or the severity of situational cues). In order to prevent sensitization effects, we decided to administer the SD3 (Jones and Paulhus, 2014) after rather than before the interview.

Participants will be asked to sign the informed consent form and give general demographic related information. Although it is possible that participants will find some content of the interview upsetting or disturbing, this is very unlikely (Corbin and Morse, 2003). Reporting on sensitive topics can even benefit participants as it gives them an opportunity to be heard and to express their thoughts and feelings (Corbin and Morse, 2003). Participants will also have the opportunity to request that their interviews are not used in our study and the option to withdraw at any stage. Finally, individuals will be asked if they can recommend further potential interview candidates who would be suited and willing to participate in the study while bringing a benefit to our project (i.e., snowballing procedure).

Information gathered throughout the interviews will be used to develop new and more detailed questions to be added to the interview guide as well as to adjust our sampling strategy by, for example, targeting additional branches (Glaser and Strauss, 1967; Eisenhardt and Graebner, 2007). Through this procedure we will be able to further verify ideas that emerged from previous interviews and to ensure that we gather a rich and comprehensive view on the situations and identify interrelations between triggers, common trends, as well as their respective validity and importance (Glaser and Strauss, 1967).

\section{Proposed Analysis}

As recommended by Corbin and Strauss (2008), data will be analyzed and discussed by multiple researchers (i.e., the authors). All interviews will be coded by pairs of two researchers to ensure 


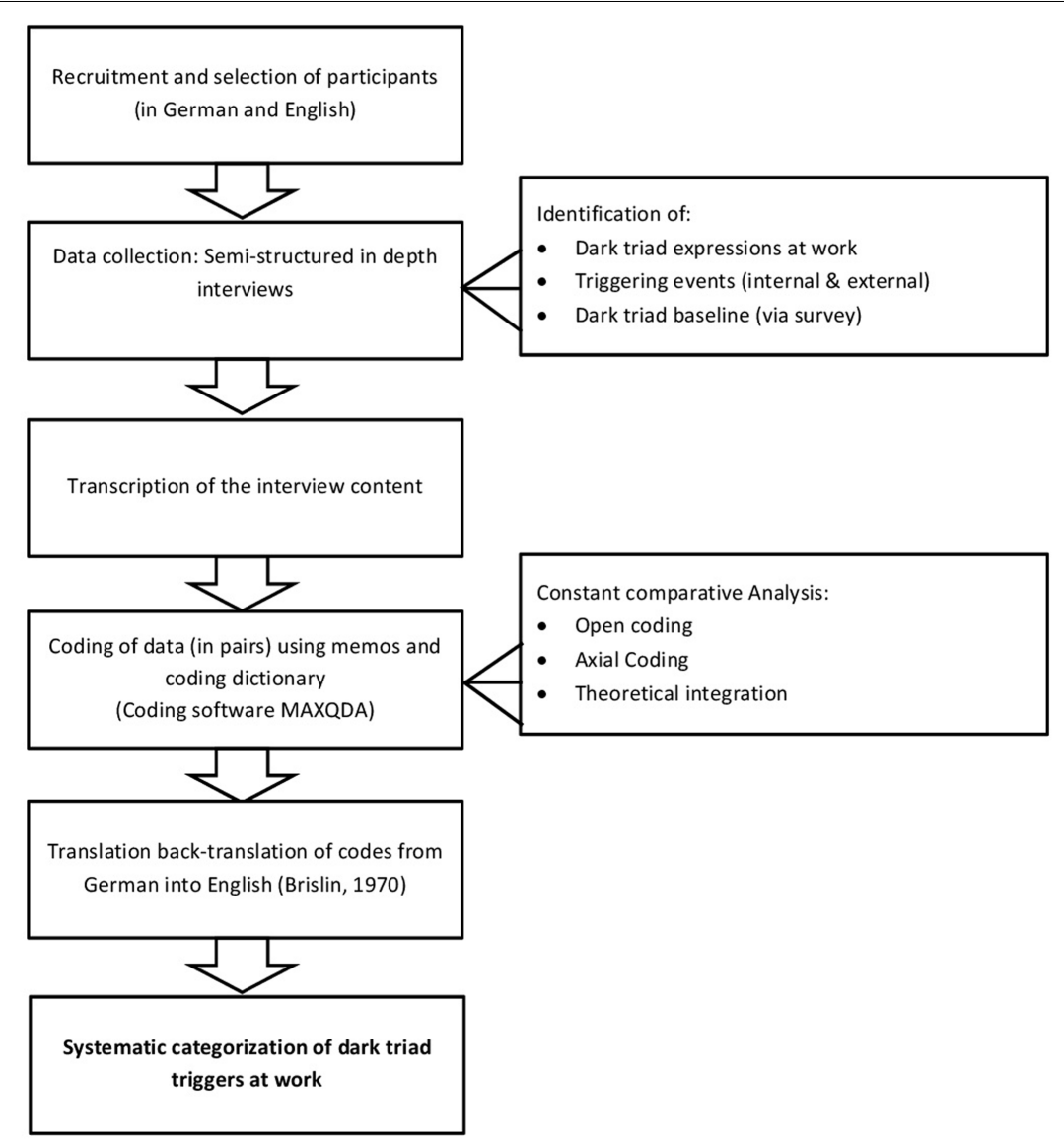

FIGURE 2 | Model of the step-by-step procedure following a grounded theory approach.

multiple perspectives on the data (Corbin and Strauss, 2008) while minimizing personal bias and increasing reliability of our coding procedure. For the coding of the transcripts we will use the coding software (MAXQDA, 1995-2017). The transcriptions will be coded in three different stages - open coding, axial coding and theoretical integration (Corbin and Strauss, 2008). Coding is a procedure through which researchers create meaningful labels for sections of texts. Using coding to make sense of the data is not a step-by-step procedure, but rather a very flexible, iterative process (Rich, 2012). The different stages do not have to be followed in a strict manner but the data may take the analysis back and forth until theoretical saturation is reached and no new coding categories emerge (Pandit, 1996; Strauss and Corbin, 1998). In order to facilitate the coding procedure, we will make use of a so-called coding dictionary, a document including the evolving system of categories that will be continually modified through constant comparative analysis of existing and newly evolving codes (Kreiner et al., 2009). In order to maintain a high theoretical sensitivity, it is essential not to emerge oneself in the existing literature, as this may lead to biased results; researcher may unconsciously search for specific information to "fit" previous findings and fail to identify other important concepts inherent in the data (Strauss and Corbin, 1998).
The first stage of coding is open coding where the data is fractured or broken down into discrete parts which are closely examined and compared and then provided with codes which at a later stage can be grouped into categories (Glaser and Strauss, 1967). In general, codes can be given to the data wordby-word, phrase-by-phrase, sentence-by-sentence, line-by-line, or paragraph-by-paragraph (Strauss and Corbin, 1998). We will use line-by-line coding which allows researchers to be an active reader while writing memos on particularly interesting codes (Strauss and Corbin, 1998; Charmaz, 2014). Memos are written records of the researchers thought process and will be taken throughout the entire study (Birks and Mills, 2015). They are essential to the discovery of the theory as they keep track of and explain the thought process of the researcher during the coding process (e.g., why the data was coded in a certain way; Birks and Mills, 2015). In order to establish consensus on the proper use of a code, pairs of coders will meet up to compare their individual codings and discuss potential discrepancies.

In the second stage we will use axial coding in order to identify links and relationships between the concepts and in order to create main and subcategories of the codes (Pandit, 1996). Explanatory and conceptual patterns and relationships are identified by looking for recurring phenomena, incidents, 
actions and interactions and by putting them in either main or subcategories (Strauss and Corbin, 1998). For example, all violence related codes could be categorized with the main code violence while subcategories could be named behavioral violence and verbal violence. In order to achieve consensus also on the more abstract categories or concepts, pairs of coders will once more meet to discuss their reasoning and approach of categorization. The emergence of new categories or changes in categories as well as their potential relation to existing literature (Locke, 2001) will be documented in the coding dictionary. Constantly comparing the data and the codes during the coding process ensures that the codes are congruent (Strauss and Corbin, 1998). In order to further explain the relationships between the concepts, we will start writing a story line, "a strategy for facilitating integration, construction, formulation and presentation of research findings through the production of a coherent grounded theory" (Birks and Mills, 2015, p. 176).

The final stage is the one of theoretical integration. In this stage a core category will be identified and the theory will be consolidated (Corbin and Strauss, 2008). A core category is defined by its ability to include all codes, sub and main categories, tying everything together to discover the theory (Corbin and Strauss, 2008). In this integrative process the storyline is developed further and results in a theory that is grounded in the data. In this process it is important to not have any preconceptions about the results and to look at the data objectively (Corbin and Strauss, 2008). Also in this final stage, pairs of coders will aim to reach consensus and will document all core categories and identified links in the coding dictionary.

\section{Ethics Statement}

This study (ECP-164_14_03_2016) has been approved by the Ethical Review Committee Psychology and Neuroscience (ERCPN) of the Faculty of Psychology and Neuroscience of Maastricht University, The Netherlands. The review was done according to Dutch law and also in the light of the highest ethical standards in the Dutch, Anglo-American and European (Union) context.

\section{ANTICIPATED RESULTS}

As very little is known about situational triggers of dark triad states at work, we can anticipate results only based on research on influencing factors of dark triad traits (Jonason et al., 2016) and maladaptive behaviors that are clinically relevant (i.e., schema modes; Keulen-de Vos et al., 2014). Research suggests for example that antisocial behavior at work (Robinson and O'LearyKelly, 1998; Lubit, 2002), competitive environments (Greenberg, 1990) and environments that can reduce a sense of behavioral accountability (e.g., cyberspace; Nevin, 2015) could facilitate dark personality expressions at work via social learning processes. In addition, scholars suggested that dark characteristics are more likely to emerge under periods of stress because this leads to a lack of cognitive resources that are needed to inhibit these dark impulses and motives in order to fulfill social expectations (Hogan and Hogan, 2001). Furthermore, research hints to the detrimental effects of traumatic experiences at work, such as victimization, threat, manipulation, bullying, and destructive leadership, which may potentially trigger expressions of dark personality states (e.g., Jonason et al., 2012; Sharf et al., 2014; Cheang and Appelbaum, 2015; Nevin, 2015; Smith et al., 2016). This is in line with research on cluster B personality disorders (e.g., antisocial personality disorder), showing that violent and delinquent behavior can be explained by an unfolding sequence of schema modes, feelings of vulnerability and abandonment or loneliness, which then lead to violent psychopathic behaviors, such as bullying and manipulation (Keulen-de Vos et al., 2014).

In sum, we expect to derive a taxonomy of triggers that represents an initial first step for conducting further quantitative research on these dynamics. For example, based on our taxonomy scholars could conduct field studies (e.g., diary studies with a cross-sectional or lagged design) as well as laboratory experiments to verify the factor structure of the triggers that we hope to identify as well as to test their causal impact on dark personality states. Further, we also call for additional qualitative studies on this topic in order to test if our taxonomy can be identified with other samples or data sources as well (i.e., proving consistency of our findings; Leung, 2015).

\section{Limitations}

Although our study has a number of strengths, it also comes with several limitations and challenges. Firstly, grounded theory remains to some extent a subjective process bearing the risk of confirmation bias (Leung, 2015). Although subjectivity is considered an undesirable confounder in quantitative research, it is considered essential and even treasurable in qualitative research as it enriches the content of the findings (Leung, 2015). However, to make our findings more valid and generalizable (Johnson, 1997), we have interviewers/coders train their interviewing and coding skills and conduct pilot interviews, try to ensure that they are aware of the preconceptions they bring to the data coding process (Strauss and Corbin, 1998) and make our philosophical stance as transparent as possible. Importantly, the generalizability of grounded theory is partially achieved through the process of abstraction applied in the entire research process via the creation of codes, categories, and core categories (Strauss and Corbin, 1998).

Secondly, a major challenge concerns the attainment of high quality responses from participants in our interviews. As the interview topic touches the personal experiences of individuals and may also potentially explore deviant or illegal activities, it is of major importance to ensure that participants feel comfortable to report about this sensitive topic and to explicitly ensure anonymity and confidentiality (Larossa et al., 1981). We aim to build in a priori strategies for evaluating and terminating an interview should participants become severely distressed (Lee and Renzetti, 1990). These may include calling participants several days after the interview or providing them with a list of local counselors should the need arise (Corbin and Morse, 2003). In any case, we will try to make very explicit when approaching participants that speaking about this topic in the interview will not have any therapeutic implications and does not compensate seeking professional help to deal with stress or trauma. 
Finally, as interviews will be conducted in German and English it is possible that cross-lingual and cross-cultural matters may arise, for instance data or categories derived in one language may not match the information derived in the other language (Squires, 2009). Although we will follow the recommendations of several authors with regard to a proper translation procedure (Temple, 2002; Van Nes et al., 2010) it is nonetheless important to acknowledge the cultural contexts people are situated in. To ensure a high quality of the project interviewers will receive training prior to conducting the interviews so as to allow them to master their interviewing skills and to minimize personal biases (Anderson, 2010). This also includes competence in cultural awareness; in other words, to be mindful of their own culture and how this may shape the interview process (Fontes, 2008).

\section{Implications and Conclusion}

The results of our study will be of theoretical as well as of practical relevance. On a theoretical level, our study will add to research on the dark triad at work which has ignored more malleable conceptualizations of personality characteristics (i.e., personality states) until now. By investigating personality dynamics as they have occurred in a specific situation, we provide a more proximal and fine-grained perspective on dark triad expressions and their eliciting factors. Further, as research on the effect of work characteristics on personality states is still in its infancy, shedding light on dark personality dynamics at work broadens the domain of triggers and personality states that are of importance in organizational settings.

Identifying the impact of situational characteristics at work on individuals' dark personality expressions is also highly relevant on a practical level. Research has shown that dark triad traits significantly relate to workplace deviance and counterproductive work behaviors (CWBs), such as workplace aggression, theft, and absenteeism (O'Boyle et al., 2012). These

\section{REFERENCES}

Anderson, C. (2010). Presenting and Evaluating Qualitative Research. Available at: http://www.ncbi.nlm.nih.gov/pmc/articles/PMC2987281/

Bansal, P., and Corley, K. (2011). The coming of age for qualitative research: embracing the diversity of qualitative methods. Acad. Manag. J. 54, 233-237. doi: 10.5465/AMJ.2011.60262792

Beckmann, N., and Wood, R. E. (eds). (2016). Dynamic personality science. Integrating between-person stability and within-person change [Research topic]. Front. Psychol. Available at: http://journal.frontiersin.org/researchtopic/ 3551/dynamic-personality-science-integrating-between-person-stabilityand-within-person-change

Berg, B. L. (2007). Qualitative Research Methods for Social Sciences, 6th Edn. Boston, MA: Pearson Education.

Birks, M., and Mills, J. (2015). Grounded Theory: A Practical Guide. Thousand Oaks, California: Sage Publications.

Bluhm, D. J., Harman, W., Lee, T. W., and Mitchell, T. R. (2011). Qualitative research in management: a decade of progress. J. Manag. Stud. 48, 1866-1891. doi: 10.1111/j.1467-6486.2010.00972.x

Bolger, N., and Zuckerman, A. (1995). A framework for studying personality in the stress process. J. Pers. Soc. Psychol. 69, 890-902. doi: 10.1037/0022-3514.69. 5.890

Brislin, R. W. (1970). Back-translation for cross-cultural research. J. Cross Cult. Psychol. 1, 185-216. doi: 10.1177/135910457000100301 unethical organizational behaviors cause extreme damage to organizations. The Association of Certified Fraud Examiners estimated that globally, businesses suffer annual losses of U\$2.9 trillion as a result of fraudulent activity (Moore et al., 2012). Building a categorization of dark triad triggers at work that will help organizations to design interventions that can prevent people from expressing their dark impulses (e.g., through appropriate job design or placement decisions) is therefore of great (economic) value. With our work, we want to support organizations to help their employees work on their dark side, thereby significantly improving people's lives in the long run.

\section{AUTHOR CONTRIBUTIONS}

All authors listed have made substantial, direct and intellectual contributions to the work, and approved it for publication. AN came up with the idea for this project, contributed to and managed all steps of the design and writing process and took the lead in writing the protocol. JB, NB, RD, HE, EAJ, and GP contributed equally to this protocol in the areas of study design and support in manuscript writing. All co-authors are listed in alphabetical order.

\section{ACKNOWLEDGMENTS}

This research was made possible by the Junior Researcher Programme (http://jrp.pscholars.org/). We would like to thank Dr. Kai Ruggeri and Elisa Haller for their feedback and support and the whole organizing team for their effort and dedication. This article was supported by the Open Access Publishing Fund of the University of Vienna, Austria. The conduction of the project will be supported by the University Fund Limburg.

Carter, G. L., Campbell, A. C., and Muncer, S. (2014). The dark triad: beyond a 'male' mating strategy. Pers. Individ. Dif. 56, 159-164. doi: 10.1016/j.paid.2013. 09.001

Charmaz, K. (2014). Constructing Grounded Theory: A Practical Guide through Qualitative Analysis. London: SAGE.

Cheang, H. S., and Appelbaum, S. H. (2015). Corporate psychopathy: deviant workplace behaviour and toxic leaders - part one. Ind. Commer. Train. 47, 165-173. doi: 10.1108/ICT-12-2013-0086

Chen, H. Y., and Boore, J. R. P. (2009). Translation and back-translation in qualitative nursing research: methodological review. J. Clin. Nurs. 19, 234-239. doi: 10.1111/j.1365-2702.2009.02896.x

Christie, R., and Geis, F. L. (1970). Studies in Machiavellianism. New York, NY: Academic Press.

Corbin, J., and Morse, J. M. (2003). The unstructured interactive interview: issues of reciprocity and risks when dealing with sensitive topics. Qual. Inq. 9, 335-354. doi: 10.1177/1077800403009003001

Corbin, J., and Strauss, A. (2008). Basics of Qualitative Research: Techniques and Procedures for Developing Grounded Theory, 3rd Edn. Thousand Oaks, CA: SAGE.

Creswell, J. W. (1998). Qualitative Inquiry and Research Design: Choosing Among Five Traditions. Thousand Oaks, CA: Sage Publications.

Dóci, E., and Hofmans, J. (2015). Task complexity and transformational leadership: the mediating role of leaders' state core self-evaluations. Leadersh. Q. 26, 436-447. doi: 10.1016/j.leaqua.2015.02.008 
Eisenhardt, K. M., and Graebner, M. E. (2007). Theory building from cases: opportunities and challenges. Acad. Manag. J. 50, 25-32. doi: 10.5465/AMJ. 2007.24160888

Fleeson, W. (2001). Toward a structure- and process-integrated view of personality: traits as density distributions of states. J. Pers. Soc. Psychol. 80, 1011-1027. doi: 10.1037//0022-3514.80.6.1011

Fleeson, W., and Jayawickreme, E. (2015). Whole trait theory. J. Res. Pers. 56, 82-92. doi: 10.1016/j.jrp.2014.10.009

Fontes, L. A. (2008). Interviewing Clients Across Cultures: A practitioner's Guides. New York, NY: Guilford Press.

Fowler, K. A., Lilienfeld, S. O., and Patrick, C. J. (2009). Detecting psychopathy from thin slices of behavior. Psychol. Assess. 21, 68-78. doi: 10.1037/a00 14938

Funder, D. C. (2016). Taking situations seriously: the situation construal model and the riverside situational Q-sort. Curr. Dir. Psychol. Sci. 25, 203-208. doi: $10.1177 / 0963721416635552$

Glaser, B. G. (1978). Theoretical Sensitivity: Advances in the Methodology of Grounded Theory. Mill Valley, CA: Sociology Press.

Glaser, B. G., and Strauss, A. (1967). The Discovery of Grounded Theory. Chicago, IL: Aldine.

Greenberg, J. (1990). Employee theft as a reaction to underpayment inequity: the hidden cost of pay cuts. Journal of Applied Psychology 75, 561-568. doi: 10.1037/0021-9010.75.6.667

Hare, R. D. (1985). Comparison of procedures for the assessment of psychopathy. J. Consult. Clin. Psychol. 53, 7-16. doi: 10.1037/0022-006X. 53.1.7

Harms, P. D., Spain, S. M., and Wood, D. (2014). Mapping personality in dark places. Ind. Organ. Psychol. 7, 114-117. doi: 10.1111/iops.12117

Hogan, R., Curphy, G. J., and Hogan, J. (1994). What we know about leadership: effectiveness and personality. Am. Psychol. 49, 493-504. doi: 10.1037//0003066X.49.6.493

Hogan, R., and Hogan, J. (2001). Assessing leadership: a view from the dark side. Int. J. Sel. Assess. 9, 40-51. doi: 10.1111/1468-2389.00162

Johnson, J. L. (1997). “Generalizability in Qualitative Research,” in Completing a Qualitative Project, ed. J. M. Morse (Thousand Oaks, CA: SAGE Publications), 191-210. doi: 10.1604/9780761906018

Jonason, P. K., and Ferrell, J. D. (2016). Looking under the hood: the psychogenic motivational foundations of the Dark Triad. Pers. Individ. Dif. 94, 324-331. doi: 10.1016/j.paid.2016.01.039

Jonason, P. K., Icho, A., and Ireland, K. (2016). Resources, harshness, and unpredictability: the socioeconomic conditions associated with the Dark Triad traits. Evol. Psychol. 14, 11. doi: 10.1177/1474704915623699

Jonason, P. K., and Jackson, C. J. (2016). The Dark Triad traits through the lens of Reinforcement Sensitivity Theory. Pers. Individ. Dif. 90, 273-277. doi: 10.1016/ j.paid.2015.11.023

Jonason, P. K., Li, N. P., Webster, G. D., and Schmitt, D. P. (2009). The dark triad: facilitating a short-term mating strategy in men. Eur. J. Pers. 23, 5-18. doi: 10.1002/per.698

Jonason, P. K., Slomski, S., and Partyka, J. (2012). The Dark Triad at work: how toxic employees get their way. Pers. Individ. Dif. 52, 449-453. doi: 10.1016/j. paid.2011.11.008

Jones, D. N., and Paulhus, D. L. (2014). Introducing the short dark triad (SD3) a brief measure of dark personality traits. Assessment 21, 28-41. doi: 10.1177/ 1073191113514105

Judge, T. A., Simon, L. S., Hurst, C., and Kelley, K. (2013). What I experienced yesterday is who I am today: relationship of work motivations and behaviors to within-individual variation in the five-factor model of personality. J. Appl. Psychol. 99, 199-221. doi: 10.1037/a0034485

Keulen-de Vos, M. E., Bernstein, D. P., Vanstipelen, S., Vogel, V., Lucker, T. P., Slaats, M., et al. (2014). Schema modes in criminal and violent behaviour of forensic cluster B PD patients: a retrospective and prospective study. Legal Criminol. Psychol. 21, 56-76. doi: 10.1111/lcrp.12047

Kreiner, G. E., Hollensbe, E. C., and Sheep, M. L. (2009). Balancing borders and bridges: negotiating the work-home interface via boundary work tactics. Acad. Manag. J. 52, 704-730. doi: 10.5465/amj.2009.43669916

Larkin, P. J., Casterle, B. D., and Schotsmans, P. (2007). Multilingual translation issues in qualitative research: reflections on a metaphorical process. Qual. Health Res. 17, 468-476. doi: 10.1177/1049732307299258
Larossa, R., Bennett, L. A., and Gelles, R. J. (1981). Ethical dilemmas in qualitative family research. J. Marriage Fam. 43, 303-313. doi: 10.2307/351382

Lee, R. M., and Renzetti, C. M. (1990). The problems of researching sensitive topics: an overview and introduction. Am. Behav. Sci. 33, 510-528. doi: 10.1177/ 0002764290033005002

Leung, L. (2015). Validity, reliability, and generalizability in qualitative research. J. Fam. Med. Prim. Care 4, 324-327. doi: 10.4103/2249-4863.161306

Lewin, K. (1936). Principles of Topological Psychology. New York, NY: McGraw Hill. Locke, K. (2001). Grounded Theory in Management Research. London: Sage.

Lubit, R. (2002). The long-term organizational impact of destructively narcissistic managers. Acad. Manag. Exec. 16, 127-138. doi: 10.5465/ame.2002.66 40218

Martin, P. Y., and Turner, B. A. (1986). Grounded theory and organizational research. J. Appl. Behav. Sci. 22, 141-157. doi: 10.1177/002188638602200207

MAXQDA (1995-2017). (Version 12) [Computer Software]. VERBI Software $\mathrm{GmbH}$. Available at: http://www.maxqda.com

McLeod, S. A. (2014). The Interview Method. Available at: www.simplypsychology. org/interviews.html

Mischel, W., and Shoda, Y. (1995). A cognitive-affective system theory of personality: reconceptualizing situations, dispositions, dynamics, and invariance in personality structure. Psychol. Rev. 102, 246-268. doi: 10.1037/ 0033-295X.102.2.246

Mischel, W., and Shoda, Y. (1998). Reconciling processing dynamics and personality dispositions. Annu. Rev. Psychol. 49, 229. doi: 10.1146/annurev. psych.49.1.229

Moore, C., Detert, J. R., Klebe Treviño, L., Baker, V. L., and Mayer, D. M. (2012). Why employees do bad things: moral disengagement and unethical organizational behavior. Pers. Psychol. 65, 1-48. doi: 10.1111/j.1744-6570.2011. 01237.x

Morf, C. C., and Rhodewalt, F. (2001). Expanding the dynamic self-regulatory processing model of narcissism: research directions for the future. Psychol. Inq. 12, 243-251. doi: 10.1207/S15327965PLI1204_3

Morse, J. M. (2000). Determining sample size. Qual. Health Res. 10, 3-5. doi: $10.1177 / 104973200129118183$

Nelson, J. (2016). Using conceptual depth criteria: addressing the challenge of reaching saturation in qualitative research. Qual. Res. doi: 10.1177/ 1468794116679873

Nevin, A. D. (2015). Cyber-Psychopathy: Examining the Relationship between Dark E-Personality and Online Misconduct. Master's thesis, The University of Western Ontario, London, ON.

Nurjannah, I., Mills, J., Park, T., and Usher, K. (2014). Conducting a grounded theory study in a language other than English: procedures for ensuring the integrity of translation. SAGE Open 4, 1-10. doi: 10.1177/2158244014528920

O'Boyle, E. H., Forsyth, D. R., Banks, G. C., and McDaniel, M. A. (2012). A metaanalysis of the Dark Triad and work behaviour: a social exchange perspective. J. Appl. Psychol. 97, 557-579. doi: 10.1037/a0025679

Pandit, N. R. (1996). The Creation of a Theory: A Recent Application of the Grounded Theory Method. Available at: http://www.nova.edu/ssss/QR/QR2-4/pandit.html

Paulhus, D. L., and Williams, K. M. (2002). The dark triad of personality: Narcissism, Machiavellianism, and psychopathy. J. Res. Pers. 36, 556-563. doi: 10.1016/s0092-6566(02)00505-6

Raskin, R., and Hall, C. S. (1979). A narcissistic personality inventory. Psychol. Rep. 45:590. doi: 10.2466/pr0.1979.45.2.590

Rauthmann, J. F., Sherman, R. A., and Funder, D. C. (2015). Principles of situation research: towards a better understanding of psychological situations. Eur. J. Pers. 29, 363-381. doi: 10.1002/per.1994

Reis, H. T. (2008). Reinvigorating the concept of situation in social psychology. Pers. Soc. Psychol. Rev. 12, 311-329. doi: 10.1177/1088868308321721

Rich, P. (2012). Inside the black box: revealing the process in applying a grounded theory analysis. Qual. Rep. 17, 1-23.

Robinson, S. L., and O'Leary-Kelly, A. M. (1998). Monkey see, monkey do: the influence of work groups on the antisocial behavior of employees. Acad. Manag. J. 41, 658-672. doi: 10.2307/256963

Roczniewska, M., and Bakker, A. B. (2016). Who seeks job resources, and who avoids job demands? The link between dark personality traits and job crafting. J. Psychol. 150, 1026-1045. doi: 10.1080/00223980.2016.1235537

Seivwright, A. N., and Unsworth, K. L. (2016). Making sense of corporate social responsibility and work. Front. Psychol. 7:443. doi: 10.3389/fpsyg.2016.00443 
Sharf, A., Kimonis, E., and Howard, A. (2014). Negative life events and posttraumatic stress disorder among incarcerated boys with callous-unemotional traits. J. Psychopathol. Behav. Assess. 36, 401-414. doi: $10.1007 /$ s10862-013-9404-z

Smith, M. B., Craig Wallace, J., and Jordan, P. (2016). When the dark ones become darker: how promotion focus moderates the effects of the dark triad on supervisor performance ratings. J. Organ. Behav. 37, 236-254. doi: 10.1002/ job. 2038

Spain, S. M., Harms, P. D., and LeBreton, J. (2014). The dark side of personality at work. J. Organ. Behav. 35, S41-S60. doi: 10.1002/job.1894

Squires, A. (2009). Methodological challenges in cross-language qualitative research: a research review. Int. J. Nurs. Stud. 46, 277-287. doi: 10.1016/j. ijnurstu.2008.08.006

Strauss, A. L., and Corbin, J. M. (1998). Basics of Qualitative Research: Techniques and Procedures for Developing Grounded Theory, 2nd Edn. Thousand Oaks, CA: SAGE.

Sturges, J. E., and Hanrahan, K. J. (2004). Comparing telephone and face-to-face qualitative interviewing: a research note. Qual. Res. 4, 107-118. doi: 10.1177/ 1468794104041110

Suddaby, R. (2006). From the editors: what grounded theory is not. Acad. Manag. J. 49, 633-642. doi: 10.5465/AMJ.2006.22083020

Sullivan, J. R. (2012). Skype: an appropriate method of data collection for qualitative interviews? Hilltop Rev. 6, 54-60.

Suls, J., and Martin, R. (2005). The daily life of the garden-variety neurotic: reactivity, stressor exposure, mood spillover, and maladaptive coping. J. Pers. 73, 1485-1510. doi: 10.1111/j.1467-6494.2005.00356.x

Temple, B. (2002). Crossed wires: interpreters, translators, and bilingual workers in cross-language research. Qual. Health Res. 12, 844-854. doi: 10.1177/ 104973230201200610
Ten Berge, M. A., and De Raad, B. (1999). Taxonomies of situations from a trait psychological perspective: a review. Eur. J. Pers. 13, 337-360. doi: 10.1002/(sici) 1099-0984(199909/10)13:53.0.co;2-f

Van Nes, F., Abma, T., Jonsson, H., and Deeg, D. (2010). Language differences in qualitative research: is meaning lost in translation? Eur. J. Ageing 7, 313-316. doi: 10.1007/s10433-010-0168-y

Vernon, P. A., Villani, V. C., Vickers, L. C., and Harris, J. A. (2008). A behavioral genetic investigation of the Dark Triad and the Big 5. Pers. Individ. Dif. 44, 445-452. doi: 10.1016/j.paid.2007.09.007

Wilhelmy, A., Kleinmann, M., König, C. J., Melchers, K. G., and Truxillo, D. M. (2016). How and why do interviewers try to make impressions on applicants? A qualitative study. J. Appl. Psychol. 101, 313-332. doi: 10.1037/apl0000046

Willig, C. (2009). Introducing Qualitative Research in Psychology: Adventures in Theory and Method, 2nd Edn. Maidenhead: Open University Press.

Zhao, H., Zhang, H., and Xu, Y. (2016). Does the Dark Triad of personality predict corrupt intention? The mediating role of belief in good luck. Front. Psychol. 7:608. doi: 10.3389/fpsyg.2016.00608

Conflict of Interest Statement: The authors declare that the research was conducted in the absence of any commercial or financial relationships that could be construed as a potential conflict of interest.

Copyright (c) 2017 Nübold, Bader, Bozin, Depala, Eidast, Johannessen and Prinz. This is an open-access article distributed under the terms of the Creative Commons Attribution License (CC BY). The use, distribution or reproduction in other forums is permitted, provided the original author(s) or licensor are credited and that the original publication in this journal is cited, in accordance with accepted academic practice. No use, distribution or reproduction is permitted which does not comply with these terms. 